\title{
MRI IMAGE SEgmentation USING LEVEL SET METHOD AND IMPLEMENT AN MEDICAL DiAGNOSIS SYSTEM
}

\author{
Paresh Chandra Barman, Md. Sipon Miah, Bikash Chandra Singh and Mst. Titasa \\ Khatun \\ ${ }^{1}$ Department of Information \& Communication Engineering, \\ Islamic University, Kushtia-7003, Bangladesh. \\ pcbarman@gmail.com, mdsiponmiah@gmail.com, bikashsingh18@yahoo.com \& \\ titasa@gmail.com
}

\begin{abstract}
Image segmentation plays a vital role in image processing over the last few years. The goal of image segmentation is to cluster the pixels into salient image regions i.e., regions corresponding to individual surfaces, objects, or natural parts of objects. In this paper, we propose a medical diagnosis system by using level set method for segmenting the MRI image which investigates a new variational level set algorithm without re-initialization to segment the MRI image and to implement a competent medical diagnosis system by using MATLAB. Here we have used the speed function and the signed distance function of the image in segmentation algorithm. This system consists of thresholding technique, curve evolution technique and an eroding technique. Our proposed system was tested on some MRI Brain images, giving promising results by detecting the normal or abnormal condition specially the existence of tumers. This system will be applied to both simulated and real images with promising results.
\end{abstract}

KEYWORDS : MANS, PL, SUI, NLOS, and LOS.

\section{INTRODUCTION}

Today's age is the age of information technology and image processing is a rapidly growing area of information technology. Its growth has been fueled by technological advances in digital imaging, computer processors and mass storage devices. This because image segmentation is the core concept of image processing. Fields which traditionally used analog systems are now switching to digital systems, for their flexibility and affordability. Image segmentation subdivides an image into its regions of components or objects and an important tool in medical image processing. The main goal of segmentation is to divide an image into parts having strong correlation with areas of interest in the image [1]. Image segmentation is an essential tool in medical image processing and is used in various applications. For example, in medical imaging filed is used to detect multiple sclerosis lesion quantification, surgical planning, conduct surgery simulations, locate tumors and other pathologies, measure tissue volumes, brain MRI segmentation, study of anatomical structure etc. Other practical applications of image segmentation are machine vision, traffic control system, face and finger print recognition and locate objects in satellite images. Magnetic Resonance Image (MRI) is a visualization image with 
Computer Science \& Engineering: An International Journal (CSEIJ), Vol.1, No.5, December 2011

a detailed internal structure of any object. It provides comparable resolution with far better contrast resolution [8]. MRI distinguishes itself from other modalities and MRI can be applied in the volumetric analysis of muscles, heart and cancer, brain tissues such as multiple sclerosis, schizophrenia, epilepsy, cerebral atrophy etc. The level set method (LSM) is a numerical technique for tracking interfaces and shapes. The advantage of the level set method is that one can perform numerical computations involving curves and surfaces on a fixed Cartesian grid without having to parameterize these objects. It has become popular in many disciplines, such as image processing, computer graphics, computational geometry, optimization, computational fluid dynamics. No doubt, in this digital world we have to implement a medical analysis system for complex medical image such as MRI image and this is the main goal of our thesis work.

\section{BACKGROUND STUDY}

In image segmentation the level to which the subdivision of an image into its constituent regions or objects is carried depends on the problem being solved. In other words, when the object of focus is separated, image segmentation should stop [1]. The main goal of segmentation is to divide an image into parts having strong correlation with areas of interest in the image. Segmentation can be primarily classified as complete and partial. Complete segmentation results in a set of disjoint regions corresponding solely with input image objects. While in partial segmentation resultant regions do not correspond directly with input image [4]. Image segmentation is often treated as a pattern recognition problem since segmentation requires classification of pixels [2]. In medical imaging for analyzing anatomical structures such as bones, muscles blood vessels, tissue types, pathological regions such as cancer, multiple sclerosis lesions and for dividing an entire image into sub regions such as the white matter (WM), gray matter (GM) and cerebrospinal fluid (CSF) spaces of the brain automated delineation of different image components are used $[2,3]$. In the field of medical image processing segmentation of MR brain image is significant as MRI is particularly suitable for brain studies because of its excellent contrast of soft issues, non invasive characteristic and a high spatial resolution.

Level Set Method is one of the emerging image segmentation techniques for medical image segmentation. The level set method is a numerical technique for tracking interfaces and shapes. It was first introduced by Osher and Sethian to capture moving fronts in 1987 [5]. The basic idea of the level set method is to represent contours as the zero level set of an implicit function defined in a higher dimension, usually referred to as the level set function, and to evolve the level set function according to a partial differential equation (PDE) [6]. In typical $P D E$ methods, images are assumed to be continuous functions sampled on a grid. Active contours were introduced in order to segment objects in images using dynamic curves. Geometric active contour models are typically derived using the Euler-Lagrange equation. In level set formulation of moving fronts (or active contours), the fronts, denoted by $C$, are represented by the zero level set $C(t)=\{((x, y) \mid \phi(t, x, y))=0\}$ of a level set function $\phi(t, x, y)$. The evolution equation of the level set function $\phi$ can be written in the following general form [10]:

$$
\frac{\partial \phi}{\partial t}+F|\nabla \phi|=0 \quad----(1)
$$

which is called level set equation [10]. The function $F$ is called the speed function. For image segmentation, the function $F$ depends on the image data and the level set function $\phi[4]$. The advantage of the level set method is that one can perform numerical computations involving curves and surfaces on a fixed Cartesian grid without having to parameterize the object. Also, the level set method provides mathematical and computational tools for the tracking of evolving 
Computer Science \& Engineering: An International Journal (CSEIJ), Vol.1, No.5, December 2011

interfaces with sharp corners and cusps, and topological changes. They efficiently compute optimal robot paths around obstacles, and extract clinically useful features from the noisy output of images. All these make the level set method a great tool for modeling time-varying objects, like inflation of an airbag, or a drop of oil floating in water.

\section{TOOLS AND TECHNIQUES}

In this work, MATLAB simulations with the mentioned algorithm will be conducted to implement in level set method. Initial level set function will be evaluated to identify the image object. To proceed our research work, we would use following techniques to solve our problem.

- Level Set Method (LSM)

- Thresholding technique

- Morphological operation (Erosion)

- Detection technique.

\section{Segmentation Methods}

Image segmentation is generally defined as the basic image processing that subdivides a digital image $f(x, y)$ into its continuous disconnect and nonempty subset $f 1, f 2, f 3, \ldots f n$ which

provides convenience to extraction of attribute [1]. There are various image segmentation techniques that are used in medical image segmentation, some are discussed bellow.

\subsection{Region Growing}

In region growing / region merging segmentation technique pixels with similar intensities are grouped. With a pixel or group of pixels known as seeds belonging to the structure in focus, the first step of this technique is started. Pixels in small neighborhood region are examined in the next step and added to the growing region on the basis of homogeneity criterion. Until no more pixels can be adjoined to the growing regions, this step continues. Finally, the object illustration is done by all added pixels to the growing regions [4].

\subsection{Graph Cut Segmentation}

In Graph Cut Segmentation, energy minimization problems can be reduced to instances of the maximum flow problem in a graph (and thus, by the max-flow min-cut theorem), define a minimal cut of the graph).Solving the pixel labeling problem is one of the most frequent applications of energy minimization in Computer Vision. Through pixel labeling problems image restoration, segmentation, problems as stereo and motion are generalized. In general energy functions like $\mathrm{E}$ are non convex functions in large dimension spaces and hence very difficult to minimize. However, when these energy functions have special characteristics, it is possible to find their exact minimum using dynamic programming. Nonetheless, it is usually necessary to rely on general minimization techniques, in the general case, like Simulated Annealing, which can be very slow in practice [7]. A property of a graph cut $C$ is that it can be related to a labeling

$f$, mapping the set of vertices $V-\{s, t\}$ of a graph $G$ to the set $\{0,1\}$, where $f(V)=0$, if 
$V \in S$, and $f(V)=1$, if $V \in T$. A binary partitioning of the vertices of the graph is defined

through this labeling. Numerous graph techniques are existed which are exploited in image segmentation such as minimum spanning trees, shortest path, graph-cuts etc. Among all these typical graph partitioning methods graph-cuts are comparatively new and the most powerful one for image segmentation [8]. Flexible and accurate global optimization and computation efficiency are achieved with graph cuts segmentation. Graph-cut segmentation was first initiated as binary image reconstruction approach in Greig et al. 1969.

\subsection{Edge Based Segmentation}

In Edge Based Segmentation technique boundary on an image or an edge is defined by the local pixel intensity gradient. An estimation of the first order derivative of the image function is called a gradient [4]. The magnitude of the gradient for a given image $f(x, y)$ can be calculated as

$$
\|G\|=\sqrt{\left[G_{x}^{2}+G_{y}^{2}\right]}
$$

The direction of gradient is represented as

$$
D=\tan ^{-1}\left(\frac{G_{x y}}{G_{y}}\right)
$$

Here, gradients in directions $x$ and $y$ are expressed as $G_{x}$ and $G_{y}$.

Edge-based techniques are fast in computation and usually in this approach a priori information about image content is not required. The most general problem of this approach is that often the edges do not enclose the object completely. In this segmentation technique the direction and magnitude can be presented as images. A post processing step of linking or grouping edges is required to structure closed boundaries neighboring regions. To compute $G_{x}$ and $G_{y}$ first and second mask are used respectively. Finally, joining $G_{x}$ and $G_{y}$ using the mentioned equation, gradient magnitude image is obtained [2].

\section{Proposed Method}

In many situations, the level set function will develop steep or flat gradients leading to problems in numerical approximations. It is then necessary to reshape the level set function to a more useful form, while keeping the zero location unchanged. One way to do this is to perform reinitialization, a technique for periodically re-initializing the level set function to a signed distance function. Re-initialization has been extensively used as a numerical remedy in traditional level set methods. The standard re-initialization method is to solve the following re-initialization equation

$$
\frac{\partial \phi}{\partial t}=\operatorname{sign}\left(\phi_{0}\right)(1-|\nabla \phi|)
$$

Where, $\phi_{0}$ is the function to be re-initialized, and $\operatorname{sign}(\phi)$ is the sign function [3].In this paper, we present a new variational level set evolving algorithm without re-initialization [9]. It consists of an internal energy term that penalizes deviations of the level set function from a signed distance function, and an external energy term that drives the motion of the zero level set toward the desired image feature [9]. This algorithm can be computed more efficiently and implemented 
Computer Science \& Engineering: An International Journal (CSEIJ), Vol.1, No.5, December 2011

using only a very simple finite difference scheme. Meanwhile, the initial contour can be anywhere in the image and a larger time step can be used to speed up the evolution. Finally we implement a medical diagnosis system by using this variational level set method with some morphological operations especially thresholding and Erosion.

Let $\Omega \subset \mathrm{R}$ be the image domain, and $u_{0}: \Omega \rightarrow \mathrm{R}$ be a given image. Also supposing $\phi_{0}$ as the initial function, let region $\Omega_{0}$ be a subset in the image domain $\Omega$, and $\partial \Omega_{0}$ be all the points on the boundaries. Initialize $\phi$ as a binary function as:

$$
\phi_{0}(x, y)=\left\{\begin{array}{cc}
-c_{0} & (x, y) \in \Omega_{0}-\partial \Omega_{0} \\
0 & (x, y) \in \partial \Omega_{0} \\
c_{0} & \Omega-\Omega_{0}
\end{array}\right.
$$

We propose a fast one-pass segmentation algorithm which is built upon flipping the values of $\phi$ at each grid point/pixel from positive to negative or vice versa according to the rule $\mathrm{R}$, and which contains 3 main steps:

1. Initialize $\phi_{0}: \Omega \rightarrow\{-1,1\}$;

2. Advance: for each grid point: set $\phi^{n+1}(x)=-\phi^{n}(x)$

$$
\text { if } R\left(\phi^{n+1}, \phi^{n}, x\right)=1 \text {; }
$$

3. Repeat until $\phi^{n+1}=\phi^{n}$;

$$
R\left(\phi^{n+1}, \phi^{n}\right) \text { can be interpreted as the logical evaluation of the following inequality: }
$$

$$
\varepsilon\left(\phi^{n+1}, c_{1}, c_{2} ; u_{0}\right) \leq \varepsilon\left(\phi^{n}, c_{1}, c_{2} ; u_{0}\right)
$$

\section{IMPLEMENTATION}

In our thesis work, we proposed a medical diagnosis system for MRI image. To implement this system we have used this variational level set method with some other image processing operation namely thresholding and erosion. The outline of our proposed medical system is shown in Fig (1).This outline heavily carries the characteristics of a typical medical diagnosis system. Final contour of the level set method gives the decision about the image object.

In implementing the proposed system, the time step $\tau$ can be chosen significantly larger than the time step used in the traditional level set methods. We have tried a large range of the time step $\tau$ in our experiments, from 0.1 to 100.Here, $\mu$ is the coefficient of the internal (penalizing) energy term $\phi$ (phi). For example, we have used $\tau=50.0$ and $\mu=0.004$ for the image in Fig (2). From our experiments, we have found that the time step $\tau$ and the coefficient $\mu$ must satisfy $\tau \mu<\frac{1}{4}$ in the difference scheme. We also found a equation for iteration numbers using the size of image. In our experiment we observe that the equation is always satisfied.

Iter_num=n_row+ (n_col *2);

where, iter_num=number of required iterations,

n_row $=$ number of rows in the used image and

n_col=number of columns in the used image.

In our experiment we use epsilon, the parameter in the definition of smooth Dirac function, default value 1.5. 
Computer Science \& Engineering: An International Journal (CSEIJ), Vol.1, No.5, December 2011

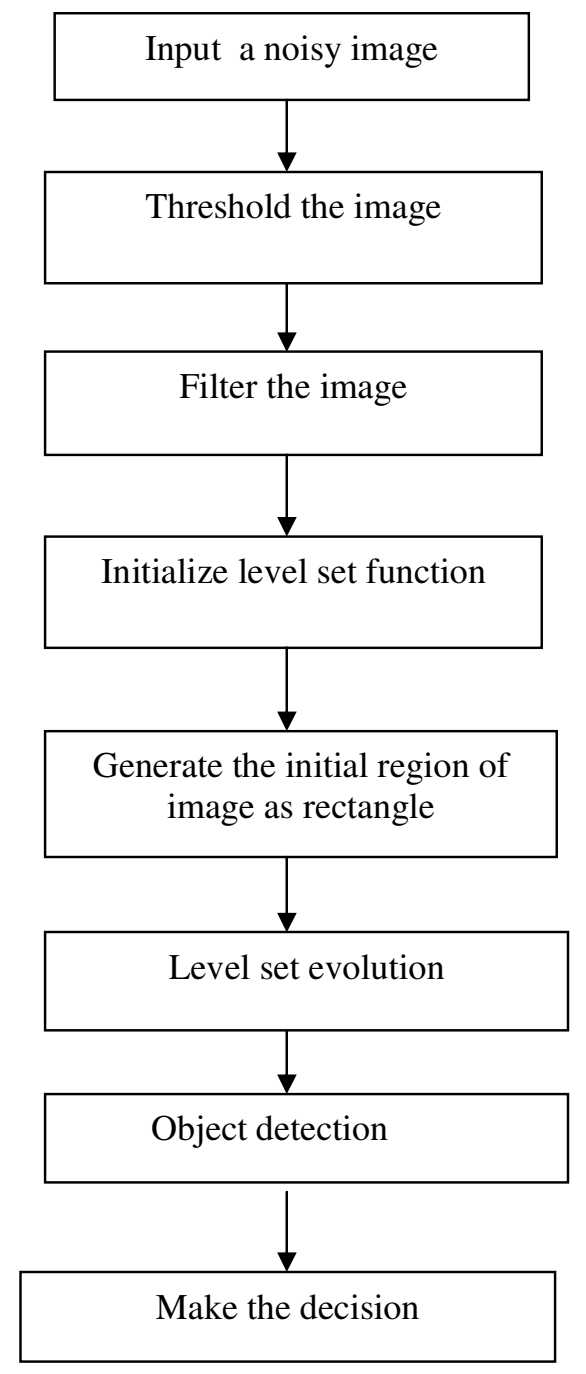

Figure 1. The outline of the proposed system

\section{EXPERIMENTAL RESULTS}

Firstly, we use a brain MRI image an abnormal condition is presence. In this MRI image, there is a tumer. In Fig (2), the input image is shown and Fig (4) shows the generated level set curve for different iteration numbers. In final contour we observe that a tumer is detected. 
Computer Science \& Engineering: An International Journal (CSEIJ), Vol.1, No.5, December 2011

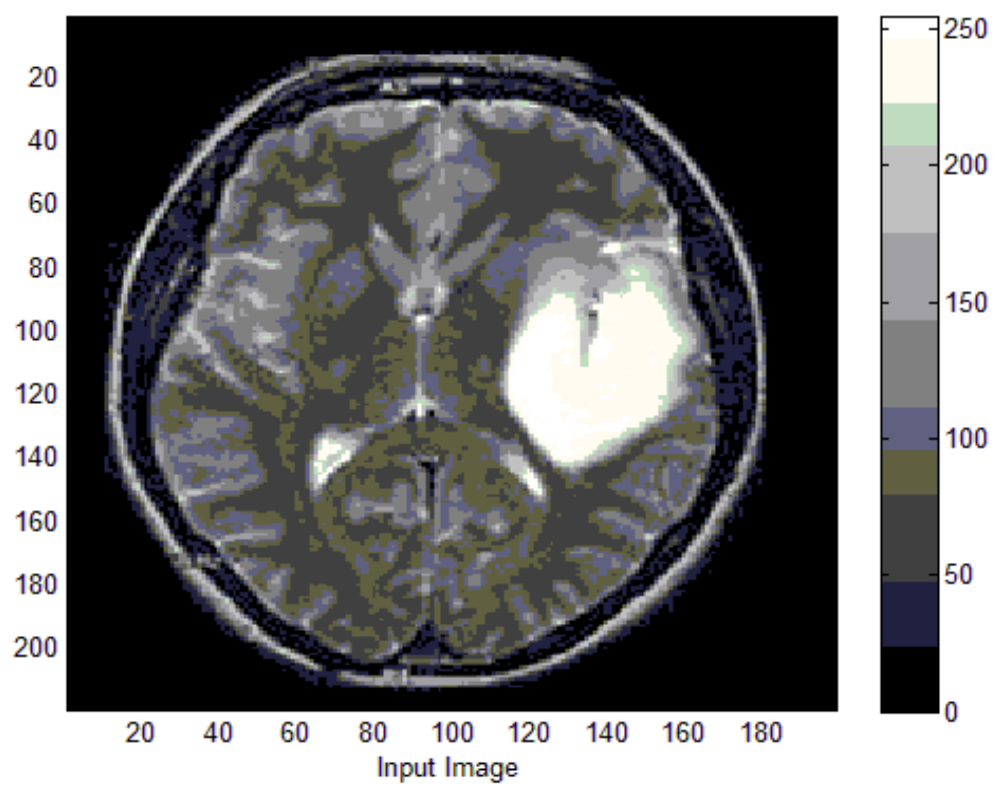

Figure 2. Input noisy image with its colorbar.

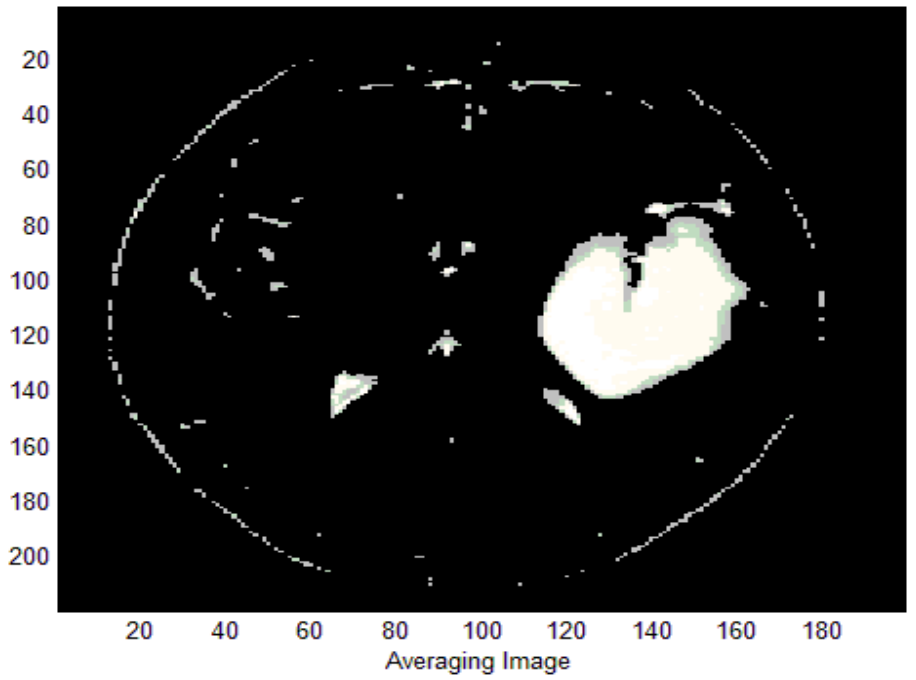

Figure 3. Average image of the input noisy image 

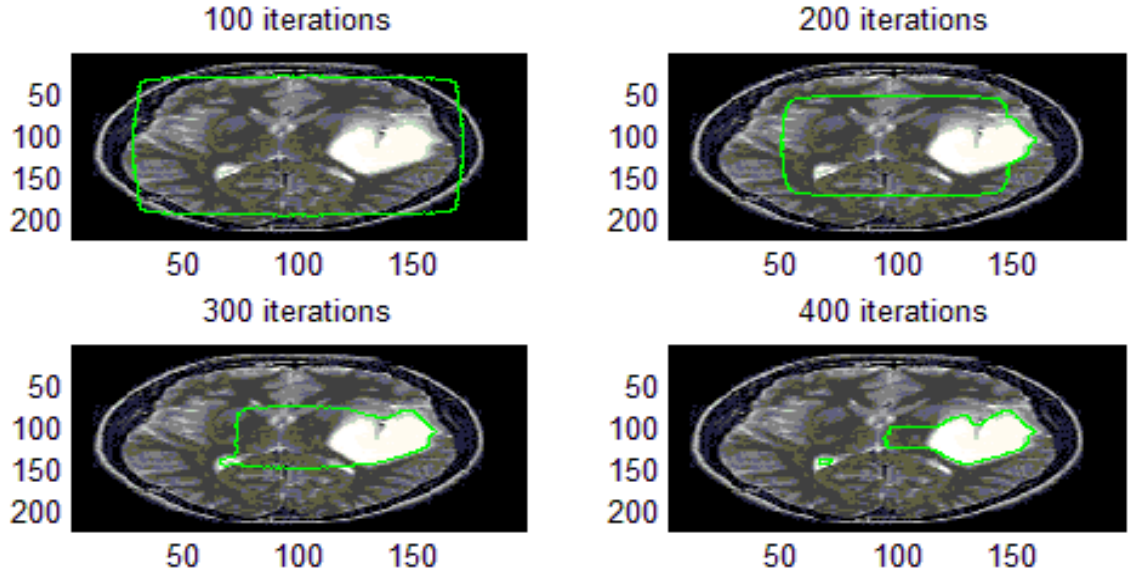

500 iterations
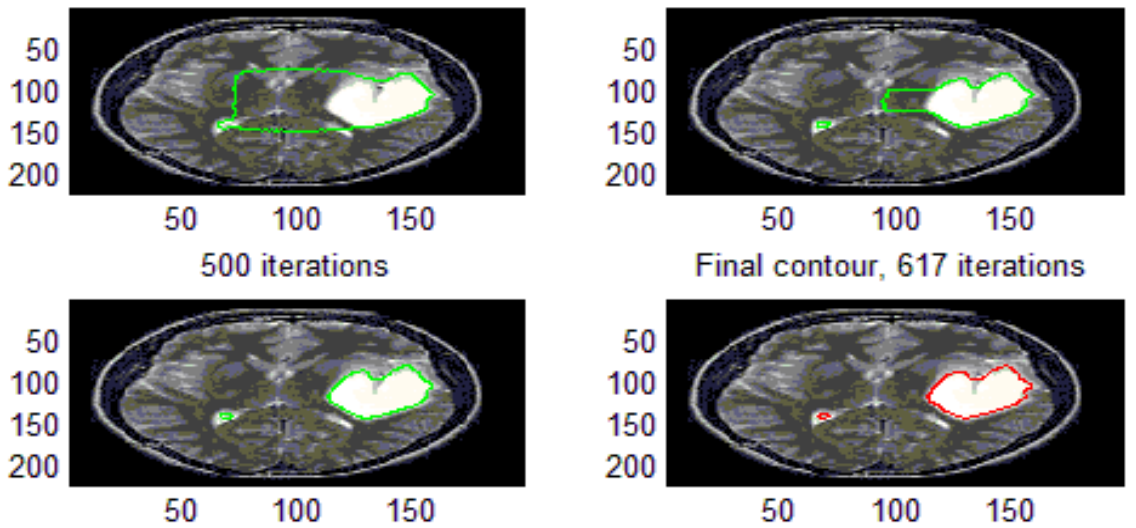

Final contour, 617 iterations

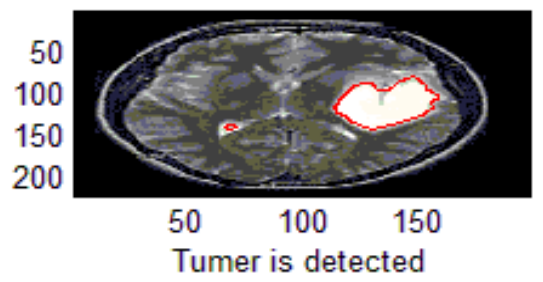

Figure 4. Results for different iterations and the final contour (Detected Tumer)

If we take a normal image as the input of our medical diagnosis system than the results of this implemented medical system are as follows:

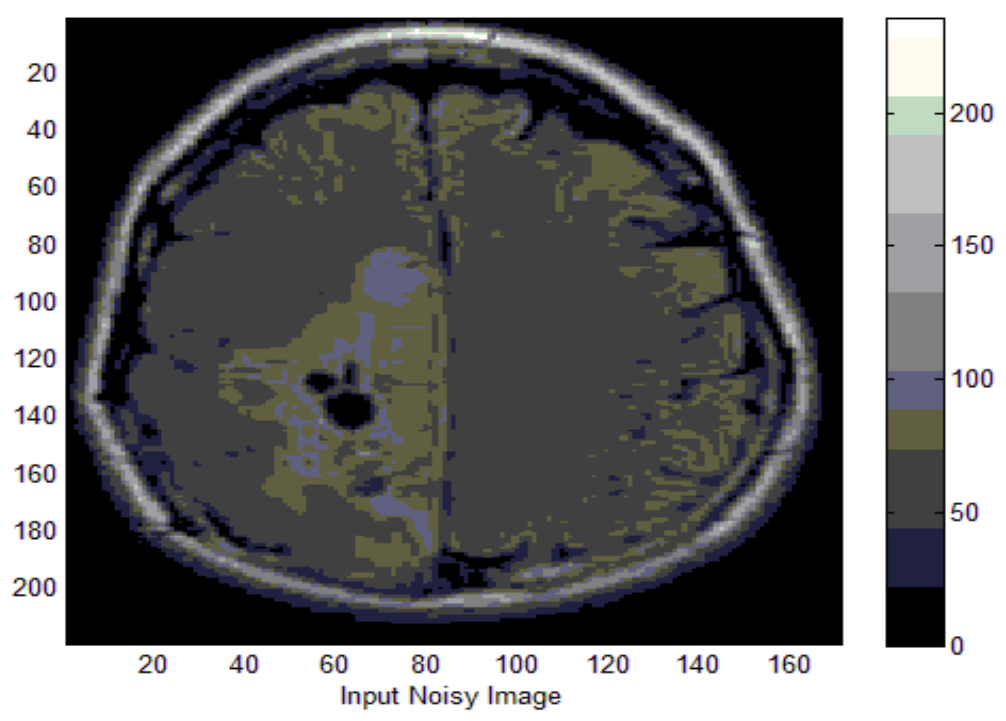

Figure 5. Input noisy image of a brain 


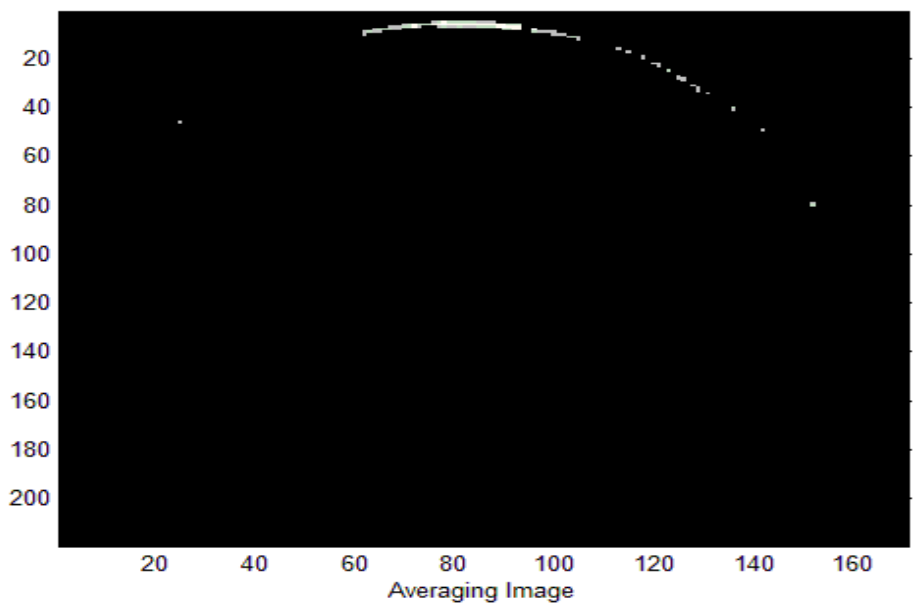

Figure 6. Corresponding average image of Fig (4).
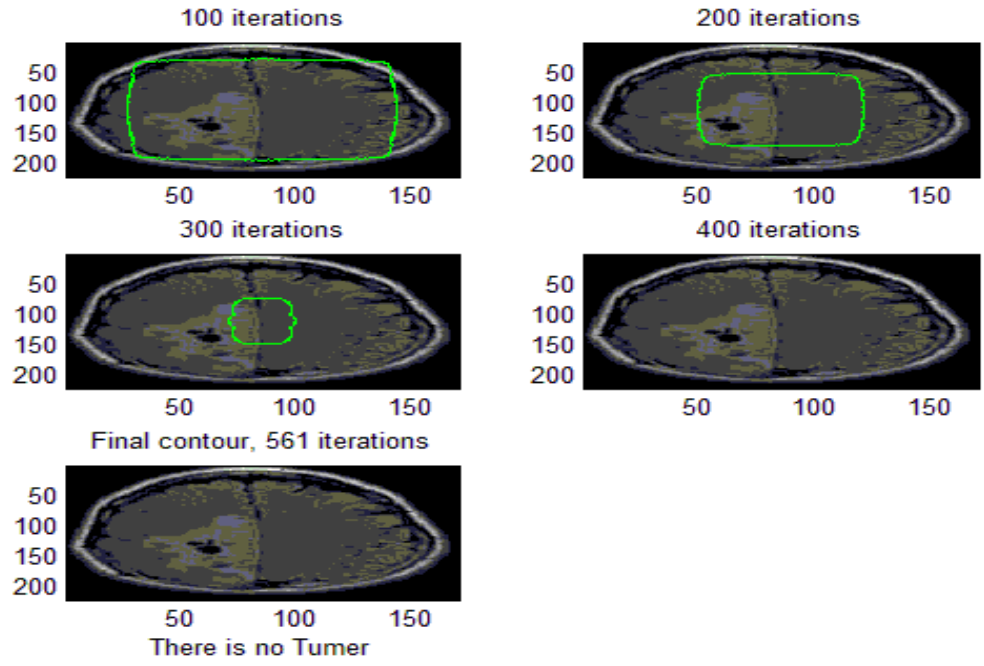

Figure 7. Results for different iterations (There is no detected Tumer)

\section{ConClusion}

In this paper we proposed a new medical diagnosis system for image segmentation. Here we used a new variational level set algorithm without re-initialization. This algorithm can be easily implemented using a simple finite difference scheme. We also used thresholding and erosion for remove the noisy element of the image. Meanwhile, not only can the initial curve be shown anywhere in the image, but the interior contours (such as tumers) can also be automatically and quickly detected. We demonstrate the performance of the proposed system and its robustness for the presence of weak boundaries and strong noise. In future, the applications of the proposed system are in the manifold image processing such as Biomedical Engineering and in computer vision. 
Computer Science \& Engineering: An International Journal (CSEIJ), Vol.1, No.5, December 2011

\section{REFERENCES}

[1] A.D. Jepson and D.J. Fleet, "Image Segmentation" 2007.

[2] C. Li, C.Y. Xu, C.F. Gui, and M.D. Fox, "Level Set Evolution without Re-initialization: A New Variational Formulation”, 2005 IEEE Computer Society Conference on Computer Vision and Pattern Recognition.

[3] Yang Fei and Jong Won Park "A New Variational Level Set Evolving Algorithm for Image Segmentation".

[4] Wen-Xiong Kang, Qing-Qiang Yang, Run-Peng Liang, "The Comparative Research on Image Segmentation Algorithms".

[5] Richard Tsal and Stanley Oshar, "Level Set Methods and Their Applications in Image Science"

[6] M. Sonka, et al. "Image processing, analysis, and machine vision", Third edition.

[7] Mahhammad Sajib Khadem, "MRI Brain Image Segmentation Using Graph Cut Methods".

[8] Issac N. Bankman, "Handbook of medical image processing and analysis", Second edition.

[9] Richard Tsal and Stanley Oshar, "Level Set Methods and Their Applications in Image Science."

[10] J. A. Sethian, Level set methods and fast marching methods, Cambridge: Cambridge University Press, 1999.

Paresh Chandra Barman has Completed his M. Sc. degree from the department of Applied Physics and Electronics, The University of Rajshahi-Bangladesh in 1995 and Ph. D. degree in Bio \& Brain Engineering, from Korea Advanced Institute of Science \& Technology (KAIST), Repub lic of Korea in 2008. He has been joined as a lecturer in the Department of Information and Communication Engineering, Islamic University, Kushtia7003, Bangladesh. Currently he is an Associate Professor and Chairman of the same department. His interested research areas are Artificial Neural Networks and Bioinformatics.

MD. SiPON MiAh has Completed his M. Sc. degree in the Department of Information and Communication Engineering from the Islamic University, Kushtia-7003, Bangladesh in 2008 and in 2010 he has been joined as a lecturer in the same department. His interested research areas are data mining, Wireless Sensor Networks, Optical fiber and Mobile communication.

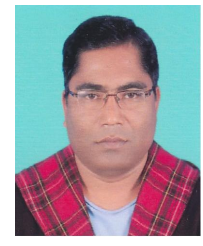

BIKASH Chandra Singh has Completed his M. Sc. degree in the Department of Information and Communication Engineering from the Islamic University, Kushtia-7003, Bangladesh in 2007 and in 2010 he has been joined as a lecturer in the same department. His interested research areas are data mining, Optical fiber and Mobile communication.

Mst. Titasa Khatun has Completed his M. Sc. degree in the Department of Information and Communication Engineering from the Islamic University, Kushtia-7003, Bangladesh in 2009. His interested research areas are wirlesscommunications. 